\title{
Mises en langage en sports collectifs : quels effets sur les apprentissages à l'école maternelle?
}

Classroom discussions within team sports: what effects on young learners?

Sandrine Prevel

\section{(2) OpenEdition}

\section{Journals}

\section{Édition électronique}

URL : https://journals.openedition.org/educationdidactique/6798

DOI : 10.4000/educationdidactique.6798

ISSN : 2111-4838

\section{Éditeur}

Presses universitaires de Rennes

\section{Édition imprimée}

Date de publication : 22 juillet 2020

Pagination : 85-97

ISBN : 978-2-7535-8121-0

ISSN : 1956-3485

\section{Référence électronique}

Sandrine Prevel, «Mises en langage en sports collectifs : quels effets sur les apprentissages à l'école maternelle? ", Éducation et didactique [En ligne], 14-2 | 2020, mis en ligne le 04 janvier 2022, consulté le 10 octobre 2022. URL : http://journals.openedition.org/educationdidactique/6798 ; DOI : https:// doi.org/10.4000/educationdidactique.6798 


\title{
MISES EN LANGAGE EN SPORTS COLLECTIFS : QUELS EFFETS SUR LES APPRENTISSAGES À L'ÉCOLE MATERNELLE?
}

\author{
Sandrine Prevel \\ Docteure en sciences de l'éducation, collaboratrice scientifique au CREN \\ (EA 2661), inspectrice de l'éducation nationale, académie de Caen \\ (sandrine.prevel1@ac-normandie.fr)
}

\begin{abstract}
Cet article s'intéresse à la manière dont des enseignants mobilisent le langage pour faire construire des savoirs spécifiques en sports collectifs en moyenne section de maternelle. Il met en relation des pratiques enseignantes et leurs effets sur les apprentissages des élèves au filtre de leurs interactions. Larticulation de trois cadres théoriques qui renvoient à l'activité de l'enseignant, à la didactique des sports collectifs et aux sciences du langage permet de construire un modèle heuristique de «mise en langage » pour rendre compte de ces pratiques. Les résultats comparent les effets produits sur les élèves par trois mises en langage différentes (technique 1, technique 2 et technique 3 ).
\end{abstract}

Mots-clés : pratique enseignante, sports collectifs, langage, école maternelle, apprentissage.

\section{Classroom discussions within team sports: what effects on young learners?}

This article investigates the ways in which teachers mobilise language to construct specific knowledge within team sports at the end of key stage 1. It relates teaching practices and their effect on pupils' learning throughout their interactions. Piecing together three theoretical frameworks relating to the teacher's actions, team sports' didactic and the science of language allows to build a "language situation" heuristic model to modelize the teaching practices. The results compare the effects on pupils via three different language situations (situation 1, situation 2, situation 3).

Keywords: teaching practices, team sports, language, primary school, learning. 


\section{INTRODUCTION}

Les travaux de recherche qui prennent pour objet le rôle du langage dans la construction des savoirs à l'école maternelle s'inscrivent le plus souvent dans des perspectives sociologiques ou sociolinguistiques. Parmi ces travaux, ceux de Bautier et Lagoueyte (2014, p. 38) mettent en évidence des pratiques dominantes centrées sur l'acquisition de « compétences techniques communicationnelles et linguistiques qui réduisent la compréhension à celle des mots ». Or, au-delà de la compréhension des mots, le langage joue un rôle essentiel dans le processus d'apprentissage. Il est même une condition d'accès aux savoirs disciplinaires, à leur formalisation et à leur partage (Astolfi, 1992). Par ailleurs, les travaux de Bautier (2001, p. 117-161) et d'autres avant elle tel Bernstein par exemple, ont montré une corrélation entre la difficulté scolaire de certains élèves issus des milieux les plus populaires et leurs pratiques langagières familiales éloignées des attendus scolaires quelles que soient les disciplines.

Nous nous intéressons ici à la manière dont des enseignantes de moyenne section mobilisent le langage pour faire construire à des élèves de moyenne section de maternelle des savoirs spécifiques en sports collectifs. Dans une approche vygotskienne de l'apprentissage, nous nous inscrivons dans une conception de l'Education Physique et Sportive (EPS) qui vise la transformation d'une motricité ordinaire en une motricité extraordinaire, dépassant les réponses corporelles spontanées. Cette transformation passe par une reconfiguration de la manière dont l'élève mobilise ses ressources et nécessite des épisodes de mise à distance de l'action. Selon nous, le langage y tient une place importante. Cependant, cette question semble peu investie à l'école maternelle, qui plus est dans le domaine des activités corporelles pour lesquelles on pense communément que l'action prime, laissant dans l'ombre la question du langage.

Nous nous intéressons plus précisément aux pratiques d'enseignantes d'école maternelle et à leurs effets sur les productions langagières des élèves lorsqu'elles traitent des objets de savoir en sports collectifs. Selon nous, certaines techniques ${ }^{1}$ mises en œuvre dans les classes favorisent un double mouvement d'apprentissages disciplinaires et d'apprentissages langagiers. À partir d'un travail de comparaison, nous identifierons des conditions favorables à l'étude de contenus disciplinaires dans les temps de classe où le langage occupe une place prépondérante et l'entrée progressive dans un mode de parler/penser/agir propre aux sports collectifs dès la maternelle.

Nous proposons des éclairages théoriques pour construire un cadre permettant de penser la relation langage/premiers apprentissages tactico-techniques. Les résultats comparent trois techniques mises en œuvre par des enseignantes expérimentées et les mettent en relation avec les productions orales des élèves qui traitent des objets en sports collectifs.

\section{DES CADRES THÉORIQUES ARTICULÉS}

Notre étude impose de construire et d'opérationnaliser un cadre articulant des éléments qui renvoient à l'activité enseignante, à la didactique des sports collectifs contextualisée à l'école maternelle pour identifier les contenus disciplinaires à faire construire par les élèves, et enfin des éléments qui renvoient à la relation langage/apprentissages.

\section{Lactivité enseignante et ses spécificités}

Nous nous intéressons à l'activité de l'enseignant dans ses perspectives technique et didactique. D'abord, nous considérons qu'enseigner c'est agencer des actions concrètes, de manière originale (dimension technique) avec un objectif d'efficacité (Vérillon, 2004). Enseigner c'est aussi faire apprendre à des élèves singuliers un savoir précis et identifié en ayant un regard constant sur le processus d'apprentissage (dimension didactique) (Orange, 2014). L'activité de l'enseignant croise des savoirs professionnels et des savoirs disciplinaires. Dans cet article, nous portons notre attention sur les savoirs discinplinaires.

À la suite de Fabre (2006), nous envisageons l'activité enseignante comme la solution à un problème professionnel qui n'est pas forcément perçu et vécu comme tel. Face à ce problème, l'enseignant apporte une réponse technique individuelle et variable. Nous mettons à l'épreuve la notion de mises en langage que nous définissons comme des réponses techniques raisonnées, prenant en charge des données ${ }^{2}$ et des nécessités ${ }^{3}$, mettant en relation des choix concrets d'intervention sur 
la situation d'enseignement et un effet escompté sur l'activité d'apprentissage des élèves. Dans leur forme experte, les mises en langage ont vocation à faire vivre les contenus d'apprentissage (Lebouvier \& Lhoste, 2013) dans les échanges de la classe et à inscrire progressivement le discours dans un mode de parler/penser/agir propre à la discipline (Jaubert et al., 2012). Précisons dès maintenant que ce mode de parler/penser/agir est en prise directe avec la signification de l'activité. Les mises en langage offrent une solution possible à un problème professionnel de guidage des apprentissages.

L'enseignant initie les mises en langage et les anime. Il agit concrètement sur la situation d'enseignement/apprentissage en fonction d'une intention. L'objet des échanges, le lieu, la mobilisation ou non d'un support (et de ce dont il est porteur), le mode de regroupement des élèves, la relation entre le temps de l'action et celui de la mise en langage et enfin sa propre activité constituent les choix d'intervention concrets qu'il effectue pour traiter le problème professionnel. Les mises en langage sont des procédés didactiques dont on peut rendre compte.

\section{Quels savoirs tactico-techniques pour l'école maternelle?}

Intéressons-nous plus précisément à la dimension didactique de l'activité enseignante. Clarifier le savoir en jeu est nécessaire pour appréhender le mode de penser et d'agir propre aux sports collectifs et le contextualiser à l'école maternelle. Le paragraphe suivant définira le mode de parler.

Les sports collectifs se caractérisent par la gestion d'un rapport de forces entre deux équipes en constante évolution. Pour marquer, le joueur doit en permanence lire le jeu, décider et agir pour peser sur ce rapport entre l'attaque et la défense. Cependant, la dimension collective nécessite la construction d'un code de communication et d'action entre joueurs pour créer de la certitude chez les partenaires et de l'incertitude chez les adversaires. Ce code de communication s'exprime dans le cadre de règles fondamentales (Gréhaigne \& Caty, 2014, p. 285) garantissant l'égalité des chances et l'incertitude du résultat. Elles régissent les modalités de la marque et la spécificité des cibles ainsi que le degré de liberté d'action sur la balle et sur les autres joueurs. Ce code de communication est une construction tactico-technique.

\section{Que retenir pour l'école maternelle?}

Le bulletin officiel spécial $\mathrm{n}^{\circ} 2$ du 26 mars 2015 définit un attendu : "Coopérer, exercer des rôles différents complémentaires, s'opposer, élaborer des stratégies pour viser un but ou un effet commun ». Nous choisissons de nous concentrer sur l'opposition pour donner sens à la coopération. Ainsi, nous adaptons les situations (espace, nombre de joueurs...) et le guidage de l'enseignant vers la mise en jeu par les élèves de maternelle de savoirs spécifiques définis dans les lignes qui suivent. Dans une perspective d'apprentissages par problématisation, nous délimitons les problèmes épistémiques que de jeunes élèves sont en mesure de traiter. Comme tout joueur de sports collectifs, l'élève de maternelle doit atteindre la cible malgré la présence des adversaires. Le problème à résoudre va se situer dans le choix entre " aller directement à la cible » ou « contourner l'adversaire ». Afin de centrer les élèves sur ces aspects et de permettre une grande quantité de tentatives autour du même problème, les situations proposées à l'école maternelle sont non réversibles et les joueurs demeurent attaquants ou défenseurs tout au long de la partie selon des rôles définis à l'avance. Cette activité d'apprentissage à partir d'un problème ne peut s'envisager sans y associer une activité langagière contribuant à la conceptualisation des savoirs et à leur partage. Ces savoirs tactico-techniques articulent des processus décisionnels et des processus d'exécution (Bouthier, 1995). Ce ne sont pas de nouvelles actions à produire mais ils s'élaborent à partir de l'expérience, des tentatives mises en œuvre par les élèves dans des situations d'apprentissage, ou de nouvelles hypothèses d'action, pour dégager des principes à prendre en compte.

Dans quelle situation de très jeunes élèves peuvent-ils construire ces savoirs? Pontais (2002) propose une forme de « jeu du loup » adaptée aux très jeunes élèves et installe ce jeu traditionnel dans l'univers des sports collectifs. Des cochons (attaquants) affrontent des loups (défenseurs) dans une situation non réversible. Il s'agit pour les premiers de transporter des objets (type lego) d'un camp à l'autre sans être interceptés par leurs adversaires. Le jeu subit un traitement didactique qui lui assure une certaine filiation avec le rugby du point de vue de la gestion des règles fondamentales (grande taille de la cible, possibilité de contact entre les adversaires). Du point de vue des savoirs, il répond à une conception fonctionnelle des 
jeux collectifs, ancrée dans la gestion d'un rapport de forces entre deux équipes qui s'opposent et vise la construction de savoirs tactico-techniques à hauteur des ressources motrices, informationnelles et énergétiques des élèves de l'école maternelle.

\section{Mise en langage et apprentissages des élèves}

Les mises en langage prennent appui sur l'idée qu'« un élève [...] devient acteur dans un contexte disciplinaire donné, dont la fonction énonciative répond à un certain nombre de valeurs et de règles historiquement et culturellement élaborées. Non seulement il construit des éléments constitutifs du savoir visé, mais il apprend simultanément à mettre en œuvre des modes d'agir/parler/penser indispensables au travail de la discipline " (Jaubert, 2007, p. 18). Nous avons préalablement esquissé les savoirs visés en sports collectifs. Arrêtons-nous maintenant sur la spécificité du langage en sports collectifs.

Le langage est présent dans les pratiques sociales de sports collectifs, avant, pendant et après le match, pour anticiper ou analyser. Comment peut-on le caractériser? Il revêt un caractère technique : il est largement connecté à l'expérience et s'organise autour de l'efficacité de l'action dans un rapport entre ce qui est profitable pour son équipe et dommageable pour les adversaires (Gréhaigne, 2014, p. 19). Par ailleurs, il accompagne une pensée tactique qui utilise dans l'action, soumise aux incertitudes du jeu et à la pression du temps, les capacités, potentiels et compétences dont le joueur dispose (Gréhaigne, 2014 , p. 32). Ainsi le discours en sports collectifs traduit l'examen des choix effectués dans l'action ou pouvant l'être, et liés aux indices que le joueur saisit sur lui-même et sur les autres au regard de leur efficacité dans des configurations de jeu déterminées conjuguant temps et espace.

\section{Quel intérêt y a-t-il à exporter ce discours dans la classe? Et comment s'y prendre?}

Les formes linguistiques du langage en EPS devraient refléter sa fonction : mettre en mots l'action mais aussi les mises en relation à effectuer pour agir. Elles peuvent témoigner d'une activité d'analyse a posteriori ou d'anticipation a priori en mobilisant un lexique dédié aux espaces, au temps, aux joueurs et une argumentation orientée vers la recherche d'efficacité. Par conséquent, une forme de langage tacticotechnique gagnerait à se déployer en EPS dès la maternelle. Elle permettrait une reconfiguration des représentations de l'activité par chaque élève, organisée par l'action et inversement dans un contexte donné.

La dimension sociale des mises en langage est double. D'une part, les échanges entre pairs participent à ces reconfigurations. D'autre part, l'intervention de l'enseignant peut rendre possible ces reconfigurations en favorisant la construction d'une position énonciative $e^{4}$ de plus en plus pertinente, dès l'école maternelle.

Les mises en langage sont destinées à faire vivre les contenus d'apprentissage et ont un fort potentiel épistémique. Ces contenus sont, selon Marsenach, « les conditions que l'élève doit intégrer pour transformer son action » (1991, p. 44). Ce sont de nouvelles mises en relation, de nouvelles combinaisons d'indices pris dans le temps, dans l'espace, sur soi et sur les autres, pour produire de nouvelles actions concrètes et efficaces. Les mises en langage constituent une instance possible de construction et de formalisation de ces nouvelles mises en relation. Cependant le travail de ces contenus ne peut se faire qu'autour d'évènements particuliers qui les mettent en scène et dont le choix relève de la responsabilité de l'enseignant.

En sports collectifs, les mises en langage devraient se concentrer sur la recherche de l'efficacité et développer une argumentation dans le registre tacticotechnique. Il s'agit d'être plus performant en mettant en relation des solutions techniques et des raisons tactiques, ce qui génère le déploiement d'une argumentation. Pour Grize (1997) argumenter n'est pas le synonyme de convaincre à tout prix mais de partager des significations avec l'interlocuteur au travers de schématisations, de lui proposer une certaine vision d'un objet de discours en soulignant certaines de ses facettes et en en gommant d'autres, de partager une vision singulière d'un objet. La schématisation est le moyen de mettre en scène un objet de discours en le soumettant à différentes opérations. Les objets de discours sont liés aux contenus d'apprentissage. Les premiers objets de discours sont les joueurs : les partenaires, les adversaires et le joueur lui-même. Les seconds objets de discours renvoient aux espaces : la cible, la zone de repli et les couloirs d'accès à la cible. La balle pourrait être un troisième objet mais elle n'est pas présente dans la situation de notre étude.

Prenons un exemple en moyenne section entre deux parties de « jeu du loup » tel que le propose 
Pontais (2002). Une élève (E), qui a marqué de nombreux points en transportant des objets dans une cible (zone de la largeur de la pièce) malgré la présence de défenseurs (des loups) produit l'énoncé suivant :

E : Y avait... y'avait pas de loup au milieu et j'ai couru au milieu pa'ce que y'avait pas de loup.

Ici l'élève décrit la solution technique raisonnée, qui lui a permis d'atteindre la cible en construisant un objet de discours qui renvoie au couloir de jeu direct (au milieu) qu'elle a choisi, compte tenu des informations saisies sur la position des adversaires (y'avait pas de loups). Le raisonnement est soustendu par la nécessité implicite d'agir en prenant en compte le rapport de forces, ce qui ne va pas de soi à l'école maternelle. Les références sont proches de celles attendues dans la discipline (au milieu) mais peuvent aussi être ancrées dans un monde de fiction (les loups) et participent de la mise en scène que propose l'élève. La conjonction de subordination «parce que » marque le lien logique entre sa trajectoire et la position des adversaires et l'efficacité de son action. La reprise-reformulation de l'énoncé accentue le lien entre le processus décisionnel et le processus d'exécution.

\section{LES ASPECTS MÉTHODOLOGIQUES DE NOTRE ÉTUDE}

Précisons maintenant le contexte du recueil des données, les outils et les étapes du traitement de ces données pour mettre en relation les techniques enseignantes de mise en langage et les prémices éventuelles d'un discours tactico-technique dans les productions langagières des élèves.

\section{Le contexte de l'étude}

Notre étude s'inscrit dans le cadre d'une recherche collaborative qui a réuni neuf enseignantes expérimentées et une doctorante autour de la gestion de la dialectique langage/apprentissages en sports collectifs à l'école maternelle. En préalable, et dans le cadre de la formation continue, les enseignantes ont eu accès à des propositions didactiques telles que le jeu du loup.

Un dispositif de formation s'est déployé sur dix-huit mois et a alterné des temps d'entretiens de formation individuels ou collectifs et des temps d'expérimentations en classe de mises en langage, au cours de trois séquences de formation. Deux expérimentations par chacune des enseignantes ont été filmées : au cours de la première séquence de formation et au cours de la dernière. Les enseignantes devaient mettre en place une situation où le langage permettait aux élèves de progresser en EPS.

\section{Une démarche pour mettre en relation des techniques et des apprentissages}

La démarche d'analyse vise à mettre en relation les mises en langage et les productions langagières des élèves pour repérer des mouvements significatifs d'apprentissages. Nous avons recueilli deux types de données. Les données d'action sont produites à partir des films des moments de classe. Elles donnent accès aux choix concrets effectués par l'enseignante (lieu de la mise en langage, relation temporelle entre le temps de l'action et celui de la mise en langage, mode de regroupement des élèves, objet des échanges, activité de l'enseignante) et aux productions langagières des élèves. Elles sont complétées par des données sur l'action, recueillies au cours des entretiens de formation. Celles-ci donnent accès aux intentions didactiques des enseignantes. Nous les exprimons en termes de nécessité. Ces entretiens ont lieu à partir d'un extrait vidéo de deux minutes environ, choisi par le chercheur car jugé révélateur de la technique mise en œuvre.

La démarche utilisée pour analyser les interactions langagières des élèves vise à rendre apparents les contenus disciplinaires mis au travail ainsi que les objets de discours en construction. Nous cherchons à savoir si les mises en langage observées permettent des évolutions dans les productions langagières des élèves. À cet égard, différents outils langagiers proposés par Fillon, Orange, Peterfalvi, Rebière et Schneeberger (2004) sont mobilisés. Les extraits vidéo ont été transcrits puis examinés de manière à repérer les objets de discours, les différentes opérations qu'ils subissent ainsi que ce qui les provoque. L'activité argumentative développée par les élèves est interrogée d'un point de vue épistémique. Dans deux cas sur trois, la mise en langage est intégrée à la séance d'EPS. Elles sont relativement brèves pour que le temps de réflexion sur l'action ne réduise pas le temps de l'action. 


\section{DES RÉSULTATS CONTRASTÉS}

Trois techniques de mise en langage conçues et animées par deux enseignantes différentes dans deux classes de moyenne section au cours de la première et de la dernière séquence de formation ont été étudiées. Nous repérons deux types de mises en langage du point de vue de ce qu'elles génèrent dans l'activité langagière des élèves et ce que celle-ci nous révèle de la construction d'un discours tactico-technique porteur de savoirs spécifiques en sports collectifs.

\section{Technique 1 : la fermeture des possibles}

La technique 1 est mise en ouvre dans la salle d'EPS de l'école, entre deux parties de jeu du loup. Les scores des deux équipes ont été comparés par correspondance termes à termes des objets gagnés. La figure 1 formalise la mise en langage.

Intéressons-nous aux effets de cette technique du point de vue des productions langagières des élèves (PE 1 : enseignante 1, E : un élève non identifié, EE : plusieurs élèves, Pie, Lou, Léo, Max et Mat : prénoms d'élèves).

À partir du constat d'échec de la défense, PE1 engage les élèves à en identifier les raisons. L'objet du discours se concentre sur l'espace de jeu occupé par la défense. Il se construit, à l'initiative de l'ensei- gnante. Cet objet va subir plusieurs opérations, prises en charge par les élèves qui vont l'inscrire dans des univers différents. D'abord ancré dans le monde de la fiction (dans la forêt), cet espace de jeu occupé par la défense s'inscrit finalement dans un univers plus proche de celui des sports collectifs et plus précis (au milieu).

PE1 : Les loups n'ont jamais gagné. Là encore ce matin, c'est encore les cochons. [...] Les copains qui étaient là, qui n'ont pas joué, je vous ai demandé de regarder quoi?

$E$ : les loups

PE1 : les loups. Ils étaient où les loups pendant le jeu?

E : Dans la forêt

PE1 : où est-ce qu'ils vont dans la forêt? Pie.?

E : au milieu

PE1 : ils étaient au milieu?

PEl se saisit de « partout » employé par les élèves comme d'une opportunité pour signaler la dispersion de la défense dans l'espace de jeu. Les élèves reprennent ce thème en l'inscrivant dans le contexte matériel du terrain de sport.

E : non ils étaient partout.

PE1 : oui?

E : Partout dans le bleu aussi

PE1 : voilà

Figure 1 : formalisation de la mise en langage 1

\section{Mise en langage 1 (PE1)}

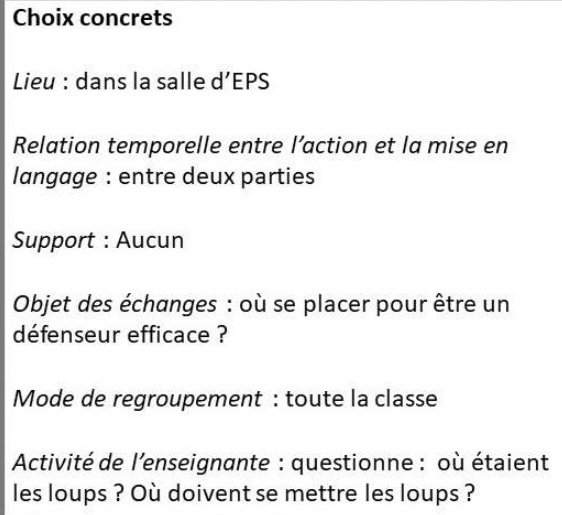

Intention didactique : faire construire la nécessité pour la défense de s'organiser collectivement

Effet escompté
Proposer une solution alternative
pour le placement de la défense


Les attaquants sont absents des échanges et rejetés par l'enseignante lorsque Léo les mentionne.

PEl poursuit son projet en interpellant individuellement les élèves.

Léo : il faut pas qu’ils sortent?

PE1 : mais attends Léo, on essaie de voir où étaient les loups ce matin. Donc ce matin les loups, ils étaient partout dans la forêt. Est-ce que tu as vu des loups partout Lou?

Lou : oui

PE1 : tu nous montres où ils étaient? Alors tu as vu des loups où? Ici. Toi tu as vu des loups où Lou? Va me montrer, où est-ce que tu as vu les trois loups?

Lou : ici

PE1 : ici! Max, où est-ce que tu as vu des loups?

Max : ici

PE1 : donc les loups ils sont partout.

PEl pose le problème de la dispersion de la défense en termes d'efficacité (peuvent) pour poursuivre le travail de la nécessité d'une organisation collective sans réellement prendre en compte l'écart à la règle signalé par un élève.

E : mais Paul il est allé dans le camp de... des cochons.

PE1 : les loups n'étaient pas dans le camp des cochons, normalement. Petit problème, quand les loups sont partout comme ça, Mél, est-ce qu'ils peuvent être après tous les cochons?

EEE : non

L'espace occupé par la défense fait l'objet d'une opération d'extension (pour empêcher...) et fait référence aux adversaires. L'enseignante cherche à faire émerger une solution alternative et collective à son placement pour permettre une plus grande incertitude du résultat. Mat propose une réponse technique (une ligne) figée qu'il évalue comme efficace (ils peuvent plus).

PE1 : alors où est-ce qu'il faudrait que les loups... on écoute, là c'est important, Clé, Mar... maintenant on va réfléchir dans sa tête fort, fort, fort.... La question est où est-ce que les loups doivent se placer dans le jeu pour empêcher les petits cochons de rapporter leur trésor?

E : je sais maîtresse.

PE1 : Mat?

Mat: il fait une ligne là et là les cochons, ils peuvent plus rentrer dans le camp.
Les interventions de PEl sont guidées par la construction de la nécessité pour la défense de s'organiser collectivement dans une conception figée de la défense où un placement organisé constituerait une solution. Cette centration sur l'organisation spatiale de la défense produit des mouvements dans la désignation des espaces et notamment dans la désignation du placement des défenseurs dans les couloirs d'accès à la cible. La solution produite par Mat ne prend pas en compte la dimension temporelle et l'évolution constante du rapport de forces. Du point de vue épistémique, le savoir ne progresse pas. Les références sont ancrées dans le monde de la fiction pour désigner les joueurs, elles sont plus diversifiées en ce qui concerne les espaces.

Les actualisations constantes du rapport de forces qui caractérisent les savoirs tactico-techniques à construire chez les élèves sont évacuées. La proposition de Mat est fortement induite par le guidage de l'enseignante qui ferme la possibilité d'explorer des possibles intégrant d'autres dimensions (temporelle, interactions avec les autres joueurs...). Si l'énoncé de Mat porte une dimension technique en proposant une solution concrète évaluée à l'aune de son efficacité (ils peuvent plus rentrer dans le camp), il porte beaucoup moins la dimension tactique propre aux sports collectifs en excluant l'inscription de la solution dans le temps.

\section{Technique 2 : L'exploration d'une solution pour créer de l'incertitude}

La mise en langage est mise en œuvre par la même enseignante dans la même classe, après une partie de jeu du loup à partir d'une maquette sur laquelle figurent les espaces de jeu et des figurines mobiles pour symboliser les joueurs. La figure 2 formalise la mise en langage (PE1 $\rightarrow$ enseignante $1, \mathrm{E} \rightarrow$ élève, Zac, Cel, Pie $\rightarrow$ prénoms d'élèves).

Intéressons-nous à cette technique du point de vue des productions langagières des élèves.

Dans cette mise en langage, PEl engage les élèves à explorer des solutions permettant aux attaquants de progresser vers la cible. Les objets de discours en construction se concentrent cette fois-ci autour des joueurs, attaquants et défenseurs. Zac propose une solution en mettant en position de thème " un cochon qui passe ». La reprise "le loup», «les loups » indique comment « ce cochon qui passe » 
Figure 2 : formalisation de la mise en langage 2

\section{Mise en langage 2 (PE1)}

Choix concrets
Lieu : dans la classe
Relation temporelle entre l'action et la mise en
langage : après l'action
Support : un support avec des figurines mobiles
Objet des échanges : Comment créer de
I'incertitude chez les défenseurs?
Mode de regroupement : un petit groupe d'élèves
dont certains ont été observateurs
Activité de l'enseignante : engage les élèves à
proposer une solution pour dégager un couloir
d'accès à la cible

Intention didactique : faire construire la nécessité de prendre en compte l'état du rapport de forces dans l'espace peut permettre une évolution du rapport de forces à un instant T propice aux attaquants. "Quand» introduit la dimension temporelle de la solution envisagée. La désignation des joueurs s'inscrit dans l'univers de la fiction.

PE1 : comment on pourrait faire pour que les cochons qui sont là puissent passer et rapporter le trésor?

Zac: moi j'attends qu'un cochon passe et quand le loup est éloigné, quand tous les loups sont éloignés...

PE1 : d'accord, tu attends qu'un autre cochon passe. Tu veux nous montrer avec le petit référentiel? Alors fais le petit cochon qui passe... Du coup, que vont faire les loups, là, si le cochon passe?

Cel. : ils vont l'attraper.

PE1 : ils vont l'attraper. Alors tu fais des loups qui attrapent le cochon.

Évacuant la proposition de Pie, PEl engage les élèves à explorer une solution possible et ses conséquences, ce qui se traduit chez les élèves par l'emploi du futur (ils vont l'attraper). Elle actualise l'objet du discours « le cochon qui passe » pour le présenter sous l'angle spatial. (il est là, les loups autour $d u$ cochon). Elle questionne sur la possibilité, pour les autres attaquants, de passer dans cette configuration. Les références restent dans l'univers de la fiction et l'enseignante prend en charge le lexique lié à l'espace.

PE1 : Là, Zac il me dit, y'a un cochon qui part. Oui, y'en a un qui part. Que vont faire les loups, là?

Pie : ils vont se mettre dans un tunnel et...

PE1 : y'a pas de tunnel là. Oui ? Cel. Tu m'as dit? Qu'est-ce qu'ils vont faire les loups là?

Cel : ils vont l'attraper.

PE1 : alors vas-y fais tous les loups qui attrapent le cochon. Le cochon il est là. On va mettre les loups. tu mets les loups autour du cochon pour qu'ils l'attrapent? Vas-y. Vas-y mets quelques loups autour du cochon. Là c'est bon, le cochon va être attrapé. Et alors là, les cochons, est-ce qu'ils peuvent passer?

E : non!

Dans ce troisième extrait, PEl met la défense en position de thème. Les opérations d'actualisation sur cet objet vont permettre à Zac d'inscrire son discours dans le traitement d'un problème concret (choisir une trajectoire en fonction des informations prises sur la défense). L'objet «loup(s)» va se construire comme pouvant être orienté (ils regardent pas) et situé dans l'espace (il est ailleurs). L'intervention de PE1 (comment on peut le faire regarder ailleurs?) contribue à présenter la défense sous l'angle de son 
orientation. Cette construction est mise en relation avec l'action des attaquants (qu'est-ce que vont faire les autres cochons?) et du temps (pendant ce temps-là). C'est sous cette forme que les contenus disciplinaires sont mobilisés pour produire une solution concrète et originale : leurrer la défense pour dégager un couloir d'accès direct à la cible et ainsi permettre la progression d'un attaquant vers la cible. La mention de «si » signale l'exploration d'une solution possible par Zac. La modalisation appréciative (ils peuvent passer en haut) indique une évaluation à l'aune de l'efficacité.

PE1 : est - ce que c'est dangereux de passer à côté les loups?

E : Mais si ils regardent pas où va le cochon.

Zac: les loups ils regardent pas où il va le cochon.

$P E 1$ : il va regarder où le loup?

Zac : il est ailleurs.

PE1 : ah, il est ailleurs? Comment on peut le faire regarder ailleurs? Comment tu pourrais faire occuper le loup pour qu'il regarde ailleurs?

Zac : si on prend quelque chose en faux, et qu'il nous touche le loup. "Non, j'ai rien dans la main" [...].

PE1 : les loups vont attraper le petit cochon qui a fait la ruse, et qu'est-ce que vont faire les autres cochons, pendant ce temps-là?

Zac: ils peuvent passer en haut.

Les interventions de PEl sont organisées par la construction de la nécessité pour les joueurs de prendre en compte l'état du rapport de forces. Les objets de discours se construisent avec l'appui de l'enseignante dans une conception des sports collectifs fondées sur un rapport de forces en évolution constante.

Dans ces extraits, Zac, propose des solutions pour peser sur le rapport de forces en créant de l'incertitude chez les défenseurs : d'abord en profitant de la sortie d'un partenaire pour progresser lui-même vers la cible puis en proposant aux adversaires un indice erroné afin de permettre à un partenaire de progresser vers la cible. Du point de vue épistémique, le savoir progresse au cours de ces extraits. La gestion du rapport de forces et les moyens pour le faire évoluer en faveur de son équipe sont au cœur des échanges. La référence est largement ancrée dans le monde de la fiction. La dimension temporelle, cruciale dans l'action est conjointement prise en charge du point de vue langagier par Zac (quand) et l'enseignante (pendant ce temps-là).
Les objets de discours liés aux attaquants et aux défenseurs se construisent successivement et vont subir des opérations qui leur donnent de la consistance sans les extraire de l'univers de la fiction.

La syntaxe de ces productions langagières est propre au langage oral, la référence reste l'univers de la fiction dans la désignation des joueurs. Le lexique lié au temps est conjointement pris en charge par l'enseignante et Zac, celui lié à l'espace majoritairement par l'enseignante. Cependant, cette mise en langage propose un projet d'action concret, raisonné qui prend implicitement en compte des savoirs tactico-techniques. Elle contient les germes d'un discours tactico-technique orienté vers l'efficacité de l'action. Elle se distingue de ce que Jaubert (2007, p. 190) nomme une chronique majoritairement organisée par la chronologie où les liens entre les éléments (but, circonstances, agents, issue) se tissent selon un modèle d'histoire.

\section{Technique 3 : Lidentification des indices} à prendre en compte pour agir

Cette dernière mise en langage est mise en ouvre entre deux parties du jeu du loup dans la salle dédiée à l'EPS. Les élèves sont regroupés en demie-classe et autour d'un tableau de scores. La figure 3 formalise la mise en langage.

Intéressons-nous à cette technique du point de vue des productions langagières des élèves ( $\mathrm{PE} 2 \rightarrow$ enseignante 2, $\mathrm{E} \rightarrow$ élève, May $\rightarrow$ prénom d'une élève).

À partir des scores obtenus par les joueurs, PE2, dans cette mise en langage, invite les élèves à repérer le plus efficace des attaquants en employant le verbe « réussir».

PE2 : bon allez on s'assoit ici, devant le tableau. Venez avec moi les trois observateurs. C'est vous qui allez m'aider à leur dire leur score. Alors, May, combien de briques a-t-elle réussi à poser dans la réserve?

EE : un, deux, trois, quatre, cinq.

PE2 : cinq briques, May a réussi à...

E : attraper!

PE2 : À attraper?

E : non, à poser!

PE2 : À poser, à apporter cinq briques dans la réserve des cochons.

EE : Lal. Un, deux, trois

PE2 : Lal. a réussi à apporter... 
EE : trois!

PE2 : trois briques sans se faire attraper, jusqu'à la réserve.

EE : (PE2 montre sur le tableau): Tho deux! Ali : deux...un.... Trois... Tim, trois...

PE2 : Qui réussit à apporter plein de briques?

EE : May!

PE2 : C'est May qui réussit à...

Un élève explique cette efficacité par une raison morphologique (parce qu'elle est grande). L'énoncé suivant de l'élève généralise par l'utilisation du pronom « on ». Pour cet élève, " courir encore plus vite » est une des conditions de l'efficacité que minimise PEl par l'emploi de « seulement». Elle met ainsi l'attaquante en positon de thème, d'abord en s'adressant à l'élève concernée puis en élargissant le questionnement aux autres élèves et en les invitant à reconsidérer (d'après vous) leur proposition.

E : parce qu'elle est grande!

PE2 : ah? tu crois que le fait qu'elle soit grande ça change quelque chose?

$E$ : parce que quand on est grand on court encore plus vite!

PE2 : Est-ce que c'est seulement parce que tu cours vite May que tu réussis?
May : oui

PE2 : si tu réussis à poser tes briques, c'est que tu ne t'es pas fait attraper.

May: non

PE2 : Alors? D'après vous si elle ne se fait pas attraper, c'est seulement parce qu'elle court vite?

Une autre explication est exprimée par un élève. La simultanéité de l'action de cette attaquante et de celle des défenseurs apparait comme une raison traduite par « en train de ». PE2 invite à examiner la possible récurrence de cette configuration (À chaque fois ?).

E : Non, c'est parce que... c'est parce que...les loups étaient en train d'attraper deux cochons.

PE2 : Les loups étaient occupés.

$E$ : À attraper deux cochons...

PE2 : À chaque fois?

May met alors le couloir d'accès direct à la cible en position de thème (au milieu) May va le présenter sous un angle temporel (temps du passé) et singulier (première personne du singulier et passé composé) pour expliquer la réussite de son projet ( $Y$ avait... y'avait pas de loup au milieu et j'ai couru au milieu pa'ce que y'avait pas de loup). PE2

Figure 3 : formalisation de mise en langage 3

\section{Mise en langage 3 (PE2)}

Choix concrets
Lieu : dans la salle d'EPS
Relation temporelle entre l'action et la mise en
langage : après une partie
Support : une affiche avec les scores
Objet des échanges : la mise en relation du choix
d'une trajectoire avec des informations prises sur le
défenseur
Mode de regroupement : en demie-classe
Activité de l'enseignante : questionne en réaction

Intention didactique : faire construire la nécessité de choisir sa trajectoire en fonction de l'état du rapport de forces 
procède à une nouvelle opération pour présenter l'objet du discours en le décontextualisant (Quand il n'y a pas de loup au milieu, elle court au milieu) en l'introduisant par "quand " et en utilisant le présent pour exprimer une mise en relation à opérer. L'articulation de processus décisionnels et de processus d'exécution est conjointement prise en charge par PE2 et une élève (choisir une bonne route) en lien avec le traitement des indices pris sur la position des défenseurs. L'emploi de « route» ancre la trajectoire dans l'univers de la fiction mais l'adjectif « bonne » indique une évaluation par l'élève dans le registre de d'efficacité.

May. : Y avait... y'avait pas de loup au milieu et j'ai couru au milieu pa'ce que y'avait pas de loup.

PE2 : Tu entends? Quand il n'y a pas de loup au milieu, elle court au milieu. Ça c'est choisir...

E : Une bonne route!

L'enjeu de la mise en langage est d'identifier des conditions favorables pour l'efficacité de l'action des attaquants. Les interventions de PE2 sont guidées par la construction de la nécessité de choisir une trajectoire en fonction de l'état du rapport de forces. Elle s'appuie sur les tentatives efficaces d'une élève. Deux objets de discours sont mis au travail : l'attaquante (elle) à l'initiative de PE2 et un second objet du discours relatif à l'espace de jeu (le milieu), mais cette fois-ci, à l'initiative d'une élève (May).

May, revient sur la solution qu'elle a mise en œuvre pour traiter le problème et aller directement à la cible en empruntant le couloir d'accès direct. Du point de vue épistémique, le savoir progresse. L'intervention de l'enseignante invite à délaisser la focalisation sur la rapidité du joueur au profit de la lecture continue d'un jeu dynamique notamment dans la relation attaquant/défenseur. Ce faisant, elle amène les élèves à mettre en relation le choix de la trajectoire avec la position, l'orientation des défenseurs et à prendre des indices sur le couloir d'accès à la cible. Parfois cet espace est obstrué par les défenseurs et parfois il est libre. C'est un élément qui pèse dans le choix de la trajectoire par l'attaquant et qui instaure la nécessité de choisir sa trajectoire en fonction du rapport de forces perçu à un instant $\mathrm{T}$ comme une raison implicite que May exprime (parce qu'il n'y avait pas de loup) dans une phrase complexe.

La référence est largement ancrée dans le monde de la fiction (une bonne route) mais le « milieu » peut renvoyer à un univers plus proche des sports collectifs. La dimension temporelle, cruciale dans l'action est prise en charge du point de vue langagier conjointement par les élèves (en train de) et par l'enseignante (quand).

Dans cet extrait, cette mise en langage met en relation un choix d'action, un résultat efficace et une raison qui prend implicitement appui sur un savoir tactico-technique. Elle développe, comme la technique précédente, les germes d'un discours tacticotechnique orienté vers l'efficacité de l'action.

\section{SYNTHÈSE DES RÉSULTATS ET DISCUSSION}

À l'issue de cette étude, il semble intéressant de revenir sur les liens entre mises en langage et productions langagières des élèves en sports collectifs à l'école maternelle. Mettons d'abord en évidence un fait saillant. Dans les trois cas, les élèves ont été placés dans des situations mobilisant au-delà des compétences techniques communicationnelles et linguistiques qui caractérisent les pratiques dominantes dans l'enseignement de l'oral (Bautier \& Lagoueyte, 2014). Pour autant, cela ne suffit pas à construire et à mettre en relation des objets de discours précis en lien avec les savoirs disciplinaires en jeu. Deux points invitent à la discussion.

\section{Un enjeu de communication en lien} avec la nature de l'activité pour les élèves

La première condition à mettre en œuvre est liée à l'activité des élèves. Dans la technique 1 , les élèves sont confrontés à un problème qui ne met pas en jeu de dimension tactique référée à la gestion du rapport de forces entre attaquants et défenseurs. L'activité langagière de l'enseignante et l'objet des échanges qu'elle cible au travers d'un questionnement fermé n'admettent des élèves que des productions langagières courtes et liées à l'espace. Ces actions langagières n'autorisent pas les mises en relation caractéristiques de la construction de savoirs tactico-techniques accompagnés d'un discours qui commence à en porter l'empreinte. Les autres choix concrets semblent relever de l'aléatoire. Les productions langagières des élèves demeurent brèves et assez peu nombreuses. Elles ne visent pas toutes la résolution d'un problème concret. 
Les techniques 2 et 3 convergent sur plusieurs points. Si l'effet escompté diffère, les élèves sont, dans les deux cas, confrontés explicitement à un problème tactico-technique soutenu par les choix de l'enseignante et notamment le support, maquette dans un cas et affiche des scores dans le second. À partir de l'affiche des scores, l'enseignante pose le problème dans la technique 3 en pointant les écarts entre les scores obtenus par les joueurs et conduit, de manière rétroactive, une élève à mettre en mots les indices qu'elle a saisis et traités pour choisir sa trajectoire. La maquette constitue une aide à l'exploration des solutions possibles dans la technique 2, qui a une visée projective. Elle permet de visualiser de manière concrète, par la manipulation des figurines dans les espaces, les réponses techniques. Dans les deux cas, plusieurs choix concrets, et notamment l'objet des échanges, convergent pour favoriser la construction par les élèves de nécessités référées à une conception des sports collectifs fondée sur la gestion d'un rapport de forces en constante évolution. Les productions langagières des élèves portent les traces de l'élaboration de ces raisonnements. Ces premiers résultats rejoignent ceux de Véjux (2017) qui montrent des dynamiques de problématisation dans des débats outillés par des maquettes en sports collectifs, chez des élèves de grande section.

\section{Des mises en langage significatives de constructions de problèmes différents}

Ce deuxième point est lié aux nécessités qui soustendent les mises en langage et qui renseignent sur le problème que construit chacune des enseignantes lorsqu'elle est amenée à guider les apprentissages de ses élèves en EPS et à les aider à se mettre à distance de l'action. Les conceptions se révèlent, notamment dans les entretiens post-séance.

Dans la technique 1, le guidage des apprentissages s'opère par un questionnement fermé qui vise à construire des techniques colorées par une conception de la défense figée ne prenant pas en compte les actualisations constantes du rapport de forces entre les équipes. Ce guidage fermé laisse peu de place à une prise en compte effective des réponses spontanées des élèves. Aucune référence n'est faite au cours de l'entretien au rôle du langage dans les apprentissages disciplinaires ou à la spécificité de l'enseignement à l'école maternelle.
Les deux autres techniques mobilisent une plus grande diversité de nécessités. Celles-ci témoignent dans les deux cas d'une conception fonctionnelle des sports collectifs et d'une conception sociale de l'apprentissage accordant une place décisive au langage, notamment pour objectiver les prestations et se mettre à distance de l'action. Ces principes fondent le choix d'un outil, la maquette, dont la fonction diffère, et orientent les interventions des enseignantes. Néanmoins, ces nécessités demeurent assez largement implicites dans les entretiens.

\section{CONCLUSION}

Notre contribution s'inscrit dans la poursuite des travaux de Jaubert, Rebière et Guillou-Kéredan (2014) qui mettent en évidence comment des élèves de petite section décalent leur pratique langagière ordinaire pour construire une position énonciative pertinente en sciences, significative d'une communauté discursive disciplinaire en germe. Notre étude explore les sports collectifs et montre des phénomènes comparables sur une tranche d'âge assez proche. Dans ce cas particulier des activités techniques corporelles, le savoir à construire se réalise dans l'action. Pour autant, le langage occupe une place dans le travail nécessaire de reconfiguration, de formalisation et de transmission qui différencie le savoir de la connaissance. Parallèlement, les productions langagières peuvent être porteuses d'indices de construction de ce savoir qui les fait évoluer. En mettant en relation les réponses techniques mises en ouvre par les enseignants avec les nécessités et les conceptions qui les fondent, les mises en langage peuvent apporter un regard nouveau sur la manière dont sont pris en compte ces éléments dans les classes.

\section{NOTES}

1. Pour Combarnous (1984) l'activité technique est une activité humaine qui mêle de l'intention et de l'utilisation. L'enseignant effectue des choix concrets pour obtenir un effet sur l'activité d'apprentissage des élèves.

2. Les données du problème sont des contraintes ou des ressources construites dans la situation (Lebouvier, 2015). 3. Les nécessités renvoient à des principes en lien avec des conceptions qui sous-tendent la pratique enseignante. 
4. Pour Jaubert (2007, p. 295) « un discours est toujours produit dans un champ de l'activité humaine, lequel a un effet sur l'énonciation elle-même. En effet l'énonciateur met en œuvre les modes de parler qu'il pense être efficaces pour élaborer les contenus nécessaires à la réussite de l'activité. Ce faisant, il se projette dans une communauté discursive dont les formes d'interactions langagières sont spécifiques, de sorte qu'il construit, au fil de l'énonciation, une position que l'on appelle une position énonciative ». Ainsi, la classe peut-être considérée comme le lieu d'essais langagiers, de brouillons.

\section{RÉFÉRENCES}

Astolfi, J.P. (1992). L'école pour apprendre. Paris : ESF éditeur.

Bakhtine, M. (1978). Esthétique de la création verbale. Paris : Gallimard.

Bautier, E. \& Lagoueyte, I. (2014). La langue et le langage en maternelle pour apprendre et devenir élève. Dans C. Passerieux (dir.), Construire le goût d'apprendre à l'école maternelle. Chronique sociale.

Bouthier, D. (1995). L'EPS et son rapport aux techniques. Spirales, 8, 96-98.

Combarnous, M (1984). Les techniques et la technicité. Messidor éditions sociales.

Fabre, M. (2006). Analyse de pratiques et problématisation : quelques remarques épistémologiques. Recherche et formation, 51, 133-145.

Fillon, P., Orange, C., Peterfalvi, B., Rebière, M. \& Schneeberger, P. (2004). Argumentation et construction de connaissances en sciences. Dans J. Douaire (dir.), Argumentation et disciplines scolaires. INRP.

Gréhaigne J.F \& Caty D. (2014). Développer la penser tactique à l'école. Dans J.F. Gréhaigne (dir.), Lintelligence tactique, des perceptions aux décisions tactiques en sports collectifs. PUFC.

Grize, J.F. (1997). Logique et langage. Ophrys.

Jaubert, M. (2007). Langage et construction de savoirs à l'école: un exemple en sciences. PUB.

Jaubert, M.,Rebiére, M.\& Berniè, J. P. (2012). Communautés discursives disciplinaires scolaires et construction de savoirs : l'hypothèse énonciative [https://hal.archivesouvertes.fr/hal-00534616].

Jaubert, M., Rebiére, M. \& Guillou-Kérédan, H. (2014). S'essayer à utiliser un texte documentaire en petite section. Repères, 50, 35-56.

Lebouvier, B., Pontais, E. (2000). Jeux sportifs collectifs de démarquage. IUFM Saint-Lô. Document interne.

Lebouvier, B. \& Lhoste, Y. (2013). Les contenus sous l'angle de la problématisation dans deux disciplines: EPS et SVT. Dans B. Daunay, Y. Reuter \& A. Thépaut (dir.), Les contenus disciplinaires, approches comparatistes. Presses universitaires du Septentrion.
Marsenach, J. et al. (1991). Éducation Physique et Sportive, quel enseignement? INRP.

Orange, C. (2014). Regard complémentaire-Unité et diversité du didactique. Éducation E Didactique, 8(1), 85-90.

Pontais, C. (2002). Jouer au loup en EPS à l'école maternelle [http://www.epsetsociete.fr/Jouer-au-loup-en-EPS].

Véjux-Grillot, M. (2017). La problématisation : une entrée pour examiner et repenser l'apprentissage des élèves en éducation physique et sportive à l'école maternelle. Recherches en éducation, 30, 63-77.

Vérillon, P. (2004). Discuter et agir pour produire des artefacts matériels en technologie au collège. Dans J. Douaire (dir.), Argumentation et disciplines scolaires. INRP. 
